\title{
Recurrent subarachnoid haemorrhage from paraventricular lesions with normal angiography
}

\author{
R. M. KALBAG
}

From the Midland Centre for Neurosurgery and Neurology, Smethwick

Vascular anomalies, either aneurysmal or arteriovenous, figure predominantly as the causative lesions in spontaneous subarachnoid haemorrhage. Such haemorrhage as the presenting feature of intracranial tumour, although much rarer, is sufficiently well recognized not to cause diagnostic difficulties; almost invariably some evidence of a space-occupying lesion, if not of a pathological circulation, is seen on subsequent angiography, especially in cases of supratentorial neoplasm. Ernsting (1955) reported a patient with recurrent subarachnoid bleeding, in whom, after carotid angiograms a year previously had been regarded as normal, a choroid plexus papilloma was demonstrated when the investigation was repeated. The lesion was confirmed by air studies and successfully removed. Ernsting pointed out the possibility of a small papilloma bleeding into the ventricle and yet not being apparent on arteriograms.

The purpose of this paper is to present three cases with recurrent haemorrhage from paraventricular lesions not revealed on angiography.

\section{CASE REPORTS}

CASE 1 T.B., a man aged 37 years, had had a subarachnoid haemorrhage in 1949 , after which he was unconscious for three weeks. He developed a left hemiplegia which improved slowly. Eventually, despite slight memory impairment, he was able to return to his former occupation as an instrument maker. In June 1958 he had a second haemorrhage, without loss of consciousness, accompanied by an increase in the left hemiparesis, which was maximal in the arm. Lumbar puncture confirmed the diagnosis.

He was admitted to the Midland Centre for Neurosurgery on 15 February 1960, when he still had a minimal spastic left hemiparesis and a complete left homonymous hemianopia. The fundi were normal.

Radiographs of the skull were normal apart from an osteoma in the right frontal sinus. Bilateral carotid angiograms (Figs. 1 and 2) were also normal. As the right posterior cerebral vessels filled on carotid injection,

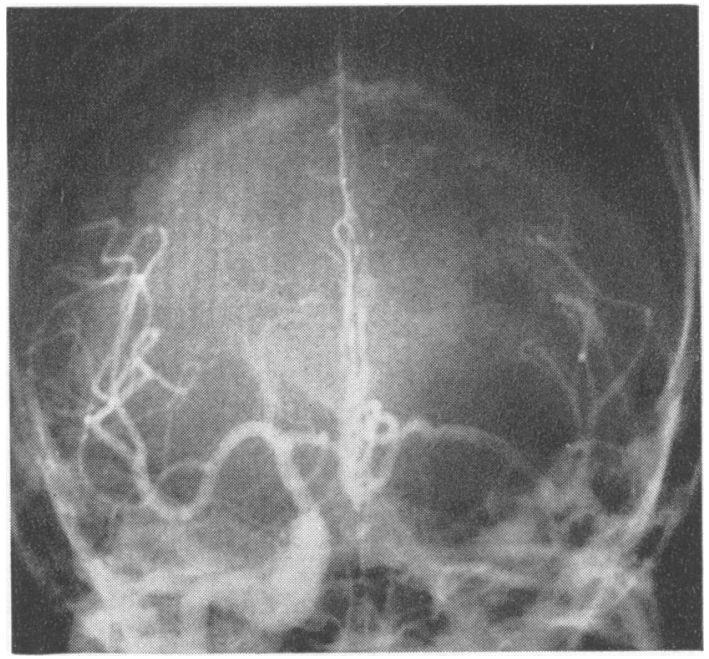

FIG. 1. Case 1: Normal antero-posterior arteriogram with compression of the left carotid artery in the neck.

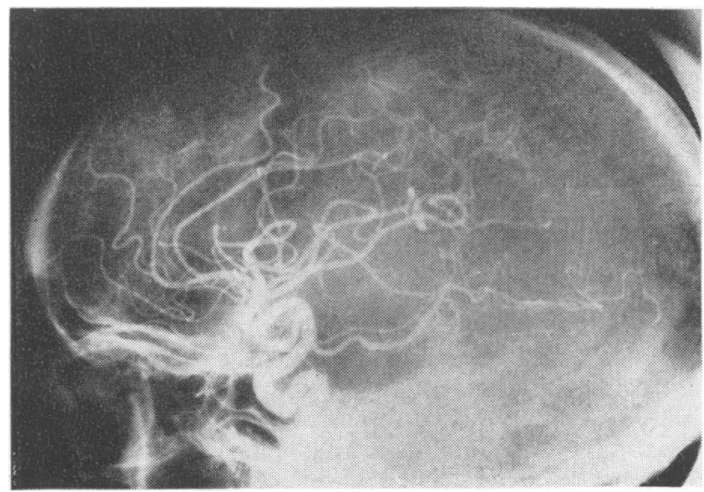

FIG. 2. Case 1: Normal lateral arterial phase on right carotid injection. 


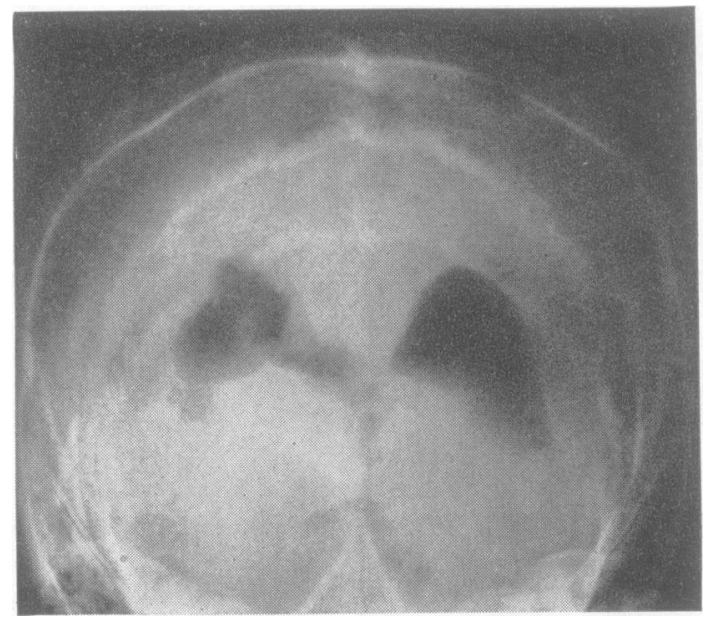

FIG. 3. Case 1: Air ventriculogram (reverse Towne's view) showing irregular filling defect in the wall of the right lateral ventricle posteriorly.

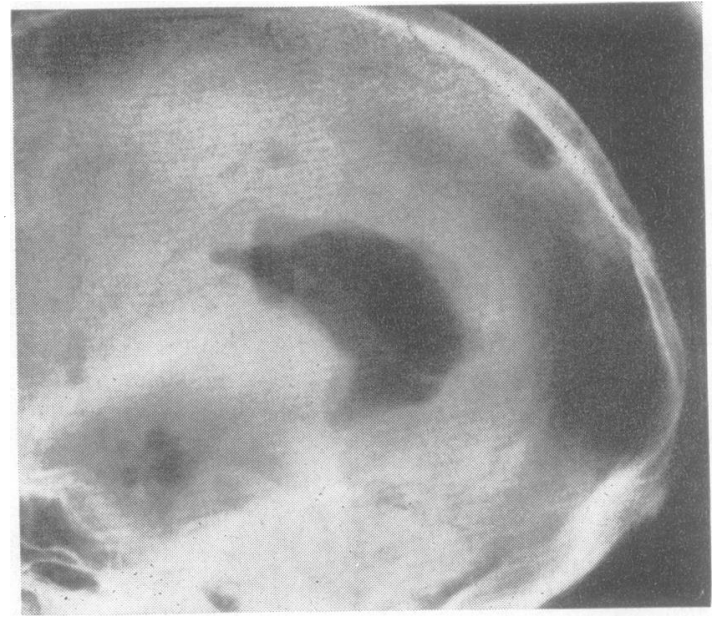

FIG. 4. Case 1: Air ventriculogram (lateral prone film) showing irregularity in the wall of the right lateral ventricle.

vertebral arteriography was considered unnecessary in the presence of the precise clinical localization of the lesion.

The cerebrospinal fluid was normal.

In view of the negative arteriography, a good prognosis was given with respect to recurrent bleeding, and two months later he returned to work.

On 13 July he was admitted to a general hospital after a fresh subarachnoid haemorrhage, when the neurological findings were again a complete left homonymous hemianopia and a left hemiplegia. There was clinical evidence of two further bleeds while he was still in hospital.
He was transferred to the Midland Centre for Neure surgery on 8 September. Bilateral carotid angiograms were repeated, and on this occasion the only abnormal findi 9 was a slight deep venous shift to the left. A right vertebol arteriogram was normal. An air ventriculogram (Figs. 3 and 4), however, showed widespread irregularities $\overline{\mathrm{c}} \mathrm{f}$ contour of the ventricular walls, maximal on the righy, posteriorly. The ventricular fluid was xanthochromic.

On 1 November an exploratory right postero-lateral craniotomy was performed by Mr. J. G. Hamilton. $\overline{\mathrm{Me}}$ found yellowish staining around the posterior end of Sylvian fissure. A core of brain was removed from the temporo-parietal region to expose soft gelatinous tissie of moderate vascularity at a depth of $1.5 \mathrm{~cm}$. continuigig down to the ventricle. On looking into the ventricle itseff, similar tissue was seen lining it in all directions. In vie of the vascularity, most of this was left undisturbed, the core of tissue obtained being sent to the laboratory. The pathologist reported that the lesion was undoubtedily an oligodendroglioma, though as tissue approached the ventricle the cells became increasingly vacuolated and degenerate.

After the operation, the patient remained unchanged neurologically, and was transferred back to the origigal hospital, where he progressively deteriorated and die few months later.

CASE 2 C.R., a girl aged 7 years, at the beginning of 1959 developed a severe headache, vomiting, andন্ণneok stiffness, without loss of consciousness. It was not c\&itgin whether this headache was of sudden onset. Fiveodays later she was admitted to a general hospital, where lumbar puncture yielded xanthochromic cerebrosp fluid. Her symptoms eased spontaneously within days.

On transfer to the Midland Centre for Neuros on 9 June 1959, apart from a possible right lower fachal weakness, there were no abnormal physical findings.

Radiographs of the skull were normal, and bilategal carotid angiography showed no convincing vascifar anomaly or displacement (Figs. 5 and 6).

After discharge, she had further episodes of recurment headache, vomiting, and neck stiffness; the cerebospinal fluid examined some days after one of these attacks was again yellow.

She was readmitted on 26 March 1960 four weeks after the last attack of headache. This time there was some blurring of disc margins with a subhyaloid haemorrîेंge on the right. On 2 April she complained of a sudden severe frontal headache, followed by vomiting, phơtophobia, and drowsiness, with marked neck retraction and a positive Kernig's sign, but no focal neurological abnormality.

Ventriculography (Figs. 7 and 8) showed a mam̂ly right frontal space-occupying lesion, indenting the anterior horn and the anterior end of the third ventriele. On 6 April a right frontal craniotomy was performed by Mr. J. M. Small, and a bluish intrinsic neoplasm with yellowish staining and a somewhat nodular capsule was totally excised.

The greater part of the specimen consisted of very lăgge blood spaces containing partly organized throm: 

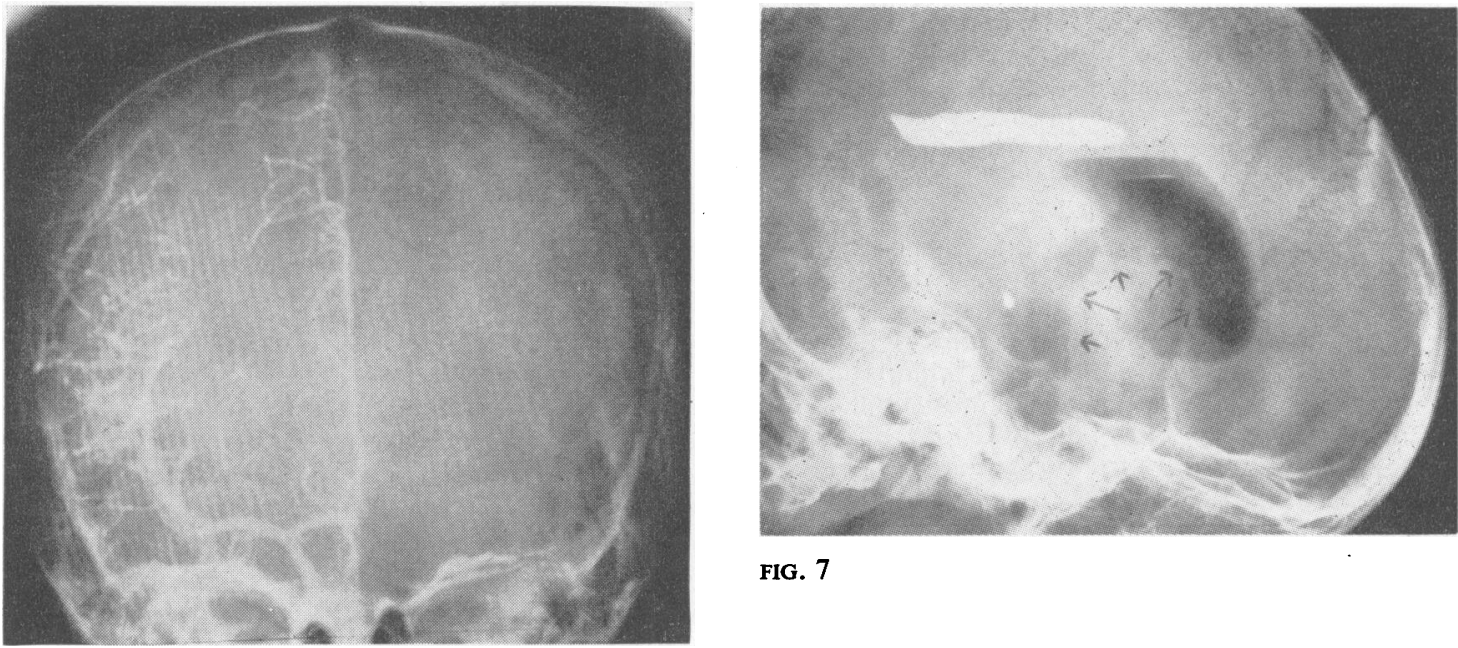

FIG. 7

FIG. 5

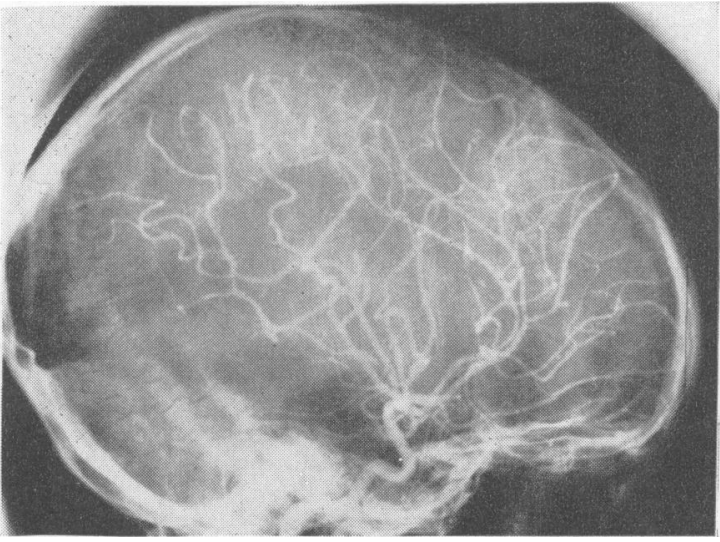

FIG. 6
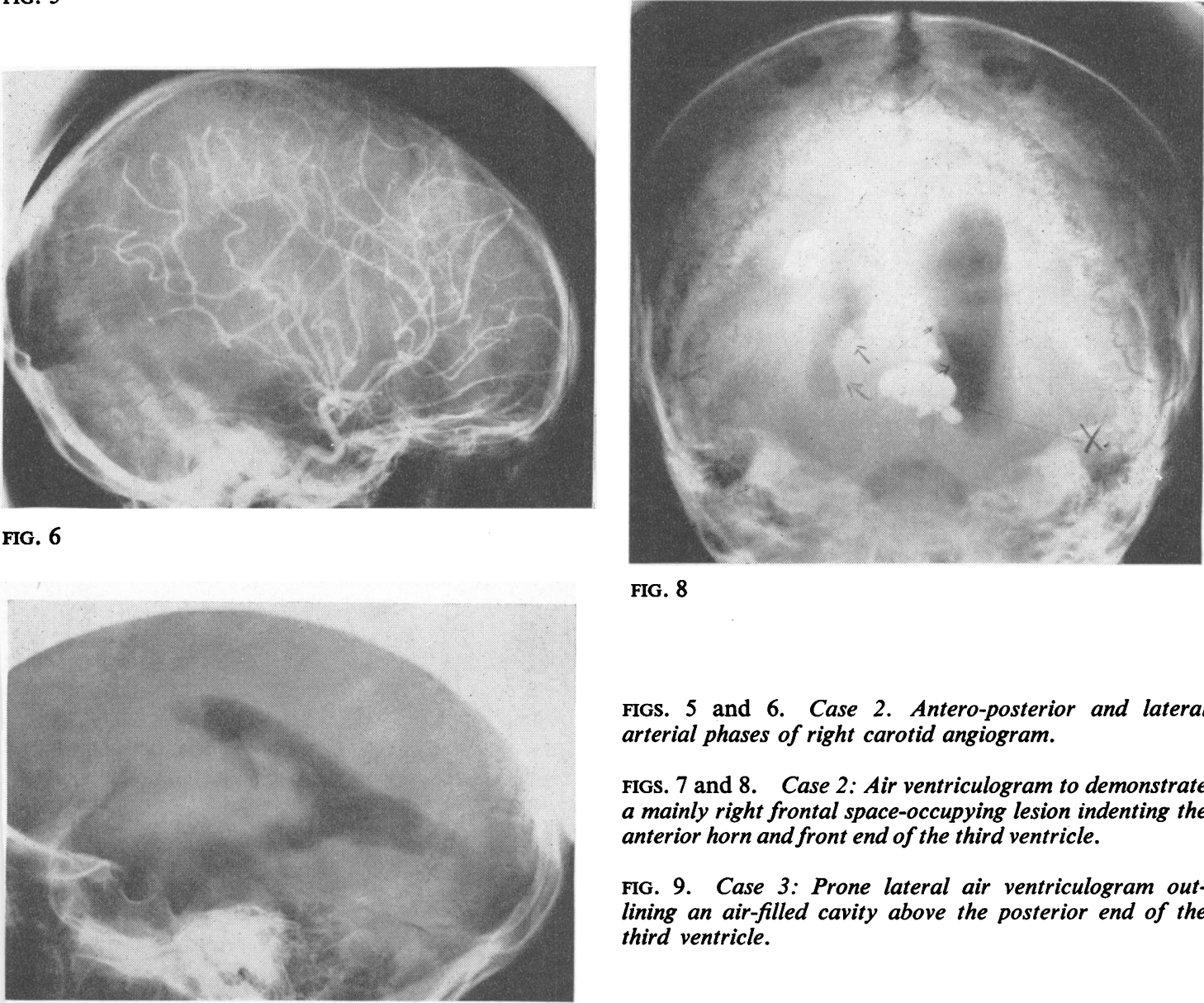

FIG. 8

FIGS. 5 and 6. Case 2. Antero-posterior and lateral arterial phases of right carotid angiogram.

FIGS. 7 and 8. Case 2: Air ventriculogram to demonstrate a mainly right frontal space-occupying lesion indenting the anterior horn and front end of the third ventricle.

FIG. 9. Case 3: Prone lateral air ventriculogram outlining an air-filled cavity above the posterior end of the third ventricle.

FIG. 9 
There was also evidence of old haemorrhage in the surrounding tissue and of a glial reaction to this. The picture was that of an angiomatous malformation rather than a neoplasm.

She was discharged home on 29 April, having recovered completely. She has remained well since.

CASE 3 L.A., a boy aged 13 years, on 28 February 1960, while out cycling with his father, developed sudden severe headache, vomited, and shortly afterwards lost consciousness. He was admitted to a general hospital deeply unconscious. Lumbar puncture yielded uniformly deeply blood-stained cerebrospinal fluid, but neck stiffness and a positive Kernig's sign were not noted till the following morning. He regained consciousness some 12 hours after admission, but remained drowsy, restless, and irritable with an increasing neck stiffness, and a pulse of $60 / \mathrm{min}$.

On 4 March he was transferred to the Midland Centre for Neurosurgery, where, apart from meningism, no abnormal neurological signs were found.

Bilateral carotid angiography showed no evidence of any supratentorial space-occupying lesion or vascular anomaly. The configuration of the anterior cerebral vessels, however, suggested enlarged lateral ventricles.

Parietal burr holes were made. Ventricular pressure was high, and the cerebrospinal fluid was stained dark brown with old blood, especially on the left side. With ventricular drainage, his headaches and level of consciousness gradually improved.

On 9 March an air ventriculogram was carried out: the ventricular size and pressure were now normal, and films showed a small space-occupying lesion with an airfilled cavity above the posterior end of the third ventricle, causing some distortion in the region of the trigone of both lateral ventricles, perhaps more so on the left (Fig. 9).

Because of the site of the lesion and the spontaneous improvement, no surgery was undertaken.

The patient remained fit and symptom free until 18 March 1962, when he felt generally unwell with an attack of 'shivering' lasting about 15 minutes, followed by severe headache and nausea without loss of consciousness. He had several such attacks in the next three days, in the course of which he also developed neck stiffness.

He was admitted to the Midland Centre for Neurosurgery on 27 June fully conscious with slight photophobia and neck stiffness and coarse horizontal nystagmus on lateral gaze, slight right lower facial weakness, bilateral extensor plantars.

On the evening of admission, he developed another severe headache with marked drowsiness, opisthotonos, and shaking of all limbs, but improved considerably after the right lateral ventricle had been tapped, releasing heavily blood-stained fluid under high pressure.

Vertebral angiography was normal. Air ventriculography again showed the small air-filled cavity in the roof of the posterior end of the third ventricle, but there was more marked generalized distension of the lateral ventricles, and no air passed into the subarachnoid space above the tentorium. Screening with Myodil confirmed that the cavity in the 'tumour' communicated with the third ventricle.
The lesion was presumed to be of a congenital nat probably a vascular hamartoma, not readily access to surgery. Following spontaneous full recovery he was discharged home on 27 July.

\section{DISCUSSION}

In all published series on subarachnoid haemorrhage, there is a proportion of cases in which no source of bleeding has been found. Björkesten and Troupp (1957), comparing the ultimate fate of 40 cases whth proven aneurysms treated conservatively, as against that of $\mathbf{6 1}$ in which no abnormality was demonstrafed on bilateral carotid arteriography, concluded that the outlook as to a fatal recurrence was 10 tines better in the latter group than in patients with known aneurysms not subjected to surgery. The proportion of negative investigations is, however, reduced when vertebral angiography has been carried out in addition. Spatz and Bull (1957) reported 60 cases, s.jn 16 of which anomalies were demonstrated on vertebral injection after having had normal bilate carotid arteriograms. It is now generally accepted that where total cerebral angiography has been normal, the likelihood of a fatal recurrence is comparatively remote.

The prognosis on the other hand is undoubme worse in the case of haemorrhage from tum\&urs. Glass and Abbott (1955) reviewed the literature on subarachnoid haemorrhage 'consequent to int fagcranial tumour'. They analysed 41 cases with recogels complete enough to be used for the study. Eatal bleeding in the initial attack was found in the instances, while in the majority of cases multiple bleeds with a fatal outcome were observed. Of their seven cases, only two had had arteriography, whish revealed the source of the bleed, while five had peictal symptoms pointing to a tumour as the proballe origin. Neoplasms lying in or alongside the ventrictes are particularly liable to present as subarachnetd haemorrhage without, at the same time, bleediog into the brain substance to form localized haematoma, and consequent vessel displacements visife arteriographically. Arterial displacement in the absence of intracerebral clot may also occ 푸, especially in the vicinity of the trigone when the tumour is large enough to obstruct the tempoial horn which later distends. In one of the two cases of $^{\circ}$ intraventricular meningioma described by Askenåy and Behmoaram (1960), while there was no vessel shift in the antero-posterior views, the middle cerebral group was raised in the lateral films. Milfer (1961) reported a case with recurrent episodes $9 f$ headache, vomiting, photophobia, and neck stiffness. in which subarachnoid haemorrhage was confirmed on lumbar puncture and the initial angiograms wofe 
normal. Some four months later following another attack and again normal angiograms, presumably because of a defect of conjugate upward movement of the eyes, an air ventriculogram was carried out to show a space-occupying lesion in the region of the pineal, which eventually proved to be a cavernous haemangioma involving the cistern of the great cerebral vein.

Fine, Paterson, and Gaylor (1960) described recurrent subarachnoid haemorrhage in the presence of an angioma associated with an intraventricular oligodendroglioma. They could not find any other instance of an oligodendroglioma bleeding spontaneously. In view of the coexistent vascular anomaly, however, it was difficult to decide with any certainty which of the two lesions was responsible for the haemorrhage.

While accepting the rare possibility of an occasional tumour being missed in cases of subarachnoid haemorrhage with normal angiograms, routine ventriculography would be impracticable and superfluous in the relatively large numbers of such cases any neurosurgical unit may have to cope with. Some attempt at establishing criteria for carrying out this procedure is therefore desirable. In this context, the term 'angiography' is deliberately preferred to 'arteriography' to emphasize an attention to all phases of the cerebral circulation. At this hospital, the investigation routinely comprises at least four views in the lateral series and three in the antero-posterior.

The presence of papilloedema or focal neurological deficit following an intracranial haemorrhage are common enough in the aneurysm group as not to justify in themselves routine ventriculography in subarachnoid haemorrhage. On the other hand, from the prognostic point alone, if for no other reason, where, in cases of recurrent subarachnoid haemorrhage, angiography has failed to reveal the source of the bleed, air studies seem to be justified in the hope of excluding the rare, sometimes operable, paraventricular neoplasm.

\section{SUMMARY}

Three cases of recurrent subarachnoid haemorrhage are reported in which angiography showed no abnormality but where lesions in or alongside the ventricles were revealed on ventriculography. It is suggested that perhaps the investigation in all instances of recurrent haemorrhage may bring to light more such tumours than has hitherto been the case.

I wish to thank Mr. J. M. Small for his interest and advice, and the consultants of the Midland Centre for Neurosurgery for permission to report on their cases.

\section{REFERENCES}

Askenasy, H. M., and Behmoaram, A. D. (1960). Subarachnoid hemorrhage in meningiomas of the lateral ventricle. Neurology (Minneap.), 10, 484-489.

Björkesten, G. af., and Troupp, H. (1957). Prognosis of subarachnoid haemorrhage. J. Neurosurg., 14, 434-441.

Ernsting, J. (1955). Choroid plexus papilloma causing spontaneous subarachnoid haemorrhage. J. Neurol. Neurosurg. Psychiat., 18, 134-136.

Fine, R. D., Paterson, A., and Gaylor, J. B. (1960). Recurrent attacks of subarachnoid haemorrhage in presence of a cerebral angioma and an intraventricular oligodendroglioma. Scot. med. J., 5, 342-346.

Glass, B., and Abbott, K. H. (1955). Subarachnoid haemorrhage consequent to intracranial tumours-Review of literature and report of 7 cases. Arch. Neurol. Psychiat., 73, 369-379.

Miller, R. H. (1961). Spontaneous subarachnoid hemorrhage: a presenting symptom of a tumor of the third ventricle. Surg. Clin. N. Amer., 41, 1043-1048.

Spatz, E. L. and Bull, J. W. D. (1957). Vertebral arteriography in the study of subarachnoid haemorrhage. J. Neurosurg., 14, 543-547. 